\title{
ChemComm
}

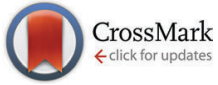

Cite this: Chem. Commun., 2017, 53,1108

Received 17th November 2016 Accepted 20th December 2016

DOI: $10.1039 / c 6 c c 09188 a$

www.rsc.org/chemcomm

\section{On the formation of concentric 2D multicomponent assemblies at the solution-solid interface $\dagger$}

\author{
Gangamallaiah Velpula, ${ }^{a}$ Takashi Takeda, $\ddagger^{\mathrm{b}}$ Jinne Adisoejoso, ${ }^{a}$ Koji Inukai, ${ }^{\mathrm{b}}$ \\ Kazukuni Tahara, $\S^{\mathrm{b}}$ Kunal S. Mali, ${ }^{* a}$ Yoshito Tobe $^{\star{ }^{\mathrm{b}}}$ and Steven De Feyter ${ }^{\star a}$
}

We report on the design and fabrication of a four-component supramolecular network using the 'core-shell' approach. Each 'core' component templates the formation of an outer 'shell' leading to formation of three concentric 'shells' around the central guest. The 'shells' are formed only in presence of guests thus demonstrating remarkable selectivity in molecular recognition.

Nanoscale materials are needed for the advancement of a number of high- as well as low-end applications. Balancing the complexity with synthetic precision at the nanometer scale however is inevitably challenging. Molecular self-assembly on solid surfaces is a promising approach in this context where organic molecules are coaxed to organize into desired crystalline patterns, typically monolayers, with periodicities at the lower end of the nanoscale. By precisely controlling the intermolecular and interfacial interactions, a wide variety of surface architectures ranging from simple lamellae to sophisticated multicomponent morphologies have been reported. The structure and composition of such nanostructured interfaces can be characterized using scanning tunneling microscopy (STM). ${ }^{1-3}$

Self-assembly becomes more and more challenging with increasing number of building blocks. Therefore, while many examples of two-component systems exist, the design and fabrication of three- or four-component supramolecular networks is rarely reported. ${ }^{4-8}$ The most popular approach is based on host-guest interactions that use size and shape recognition,

\footnotetext{
${ }^{a}$ Division of Molecular Imaging and Photonics, Department of Chemistry, KU Leuven-University of Leuven, Celestijnenlaan 200F, B-3001 Leuven, Belgium. E-mail:kunal.mali@kuleuven.be, steven.defeyter@kuleuven.be

${ }^{b}$ Division of Frontier Materials Science, Graduate School of Engineering Science, Osaka University, Toyonaka, Osaka 560-8531, Japan.

E-mail: tobe@chem.es.osaka-u.ac.jp

$\dagger$ Electronic supplementary information (ESI) available: Synthesis of DBA derivatives and additional STM data. See DOI: 10.1039/c6cc09188a

\# Present address: Institute of Multidisciplinary Research for Advanced Materials, Tohoku University, Sendai, Miyagi, 980-8577, Japan.

$\S$ Present address: Department of Applied Chemistry, School of Science and Technology, Meiji University, 1-1-1 Higashimita, Tama-ku, Kawasaki, 214-8571, Japan.
}

and complementary interactions between assembling components. ${ }^{9}$ For example, Adisoejoso et al. reported a four-component Kagomé network which was used to host three different types of guest species. ${ }^{6}$ Multicomponent systems based on non-host-guest interactions are considerably rare. A notable example is the four-component network reported by Xue et al. where the shape complementarity of alkadiyne side chains was used for building ordered networks with repeat units up to $23 \mathrm{~nm} .{ }^{10}$

The precise control over the surface stoichiometry of components is dictated by complex thermodynamic processes that define the self-assembly process itself. In fact, most design strategies are based on enthalpic considerations. While for many systems thermodynamics rules, in some cases, the nanostructured networks are kinetic in origin despite being of high structural quality. ${ }^{11}$ The relative importance of kinetics versus thermodynamics at a given temperature determines if the components self-assemble into a crystalline multicomponent network, undergo random mixing, exhibit competitive adsorption or a combination thereof. ${ }^{12,13}$

Here we describe the design and formation of a fourcomponent network using a novel strategy where the building blocks co-crystallize in a concentric fashion around a central guest leading to formation of a (laterally) layered assembly (Fig. 1). The network is formed at the organic solution-highly oriented pyrolytic graphite (HOPG) interface and characterized at sub-molecular resolution using STM. The design strategy allows formation of three concentric shells, each made up of six molecules, around the central guest. The outer shells are only formed in presence of an inner component thus exhibiting remarkably high selectivity in supramolecular recognition.

Coronene (COR) was chosen as the central guest. It acts as the ultimate core component around which the peripheral shells assemble. It is a rigid aromatic molecule with six-fold symmetry. Shells with six-fold symmetry approach the shape of a ring and the formation of supramolecular hexagons is welldocumented. ${ }^{9}$ For optimal size complementarity, a cyclic hexamer of hydrogen-bonded isophthalic acid (ISA) molecules was selected as the first shell around COR. This heteromolecular cluster is 
a)

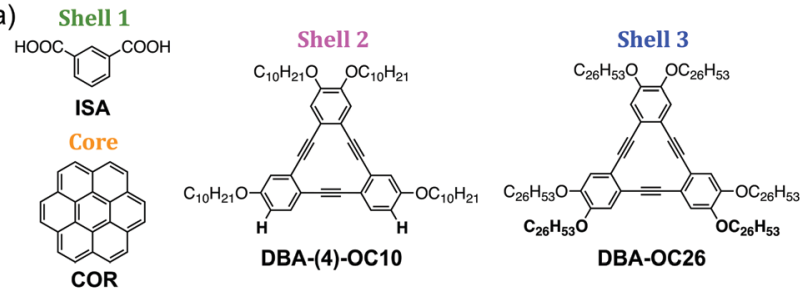

b)
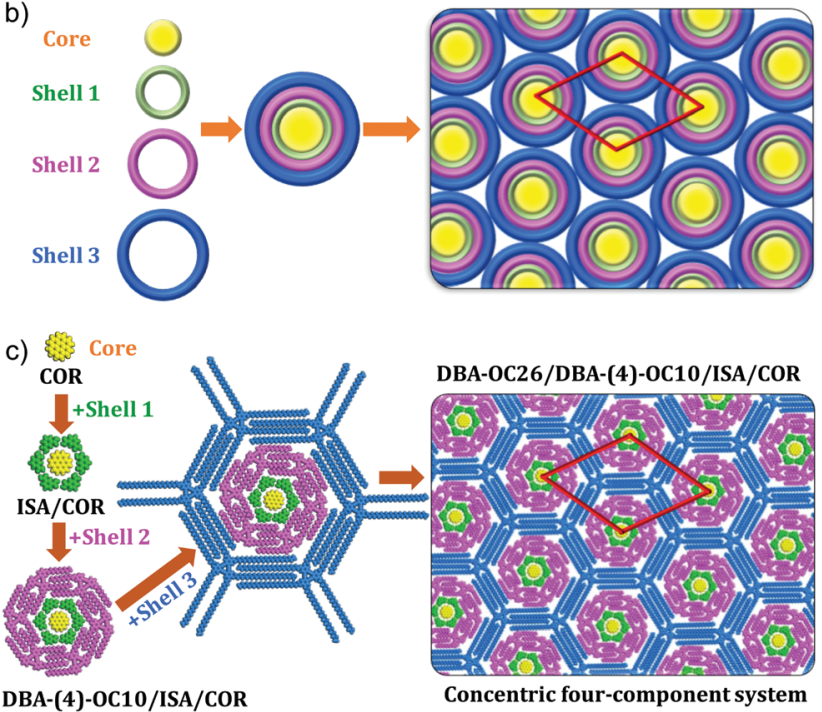

Fig. 1 (a) Chemical structures of the compounds. (b) Schematic illustration of the 'core-shell' multicomponent system in which each core-shell structure is assembled in a $p 6$ plane group. (c) Molecular models illustrating the design strategy.

stabilized by van der Waals interactions between COR and ISA. ${ }^{14}$ The absence of a third carboxyl group (as in trimesic acid, which forms an extended host-guest network with $\mathbf{C O R}^{14,15}$ ) allows the COR-ISA cluster to act as a discrete guest (see Fig. S1 in ESI $\dagger$ ).

From a design point of view, the multishell network is built up shell-by-shell, meaning that the inner diameter of the shell " $x$ " must fit the outer diameter of shell " $x-1$ ", with a precision of about $0.1 \mathrm{~nm}$. This is necessary for good van der Waals contact between the shells. The demand for dimensionality control limits the choice of molecular systems. The next shell was thus designed by considering the approximate diameter of the COR-ISA heteromolecular cluster $(c a .2 .8 \mathrm{~nm})$. We have reported a three-component system based on encapsulation of COR-ISA clusters by extended honeycomb networks formed by a dehydrobenzo[12]annulene (DBA) derivative substituted with six decyloxy chains. ${ }^{14}$ Alkoxy DBA derivatives are known to form stable nanoporous networks via interdigitation between alkoxy chains. ${ }^{16}$ A DBA derivative with decyloxy chains can serve as the second shell provided that its tendency to form extended host network by itself is prohibited (see Fig. S2 in ESI $\dagger$ ). From a design point of view, this can be achieved by replacing two alkoxy chains with hydrogen atoms such that extended honeycomb network formation is not possible. Thus, a DBA derivative with four decyloxy chains (DBA-(4)-OC10, Fig. 1a) was synthesized as the constituent of the second shell. We reasoned that this design will allow the DBA-(4)-OC10-ISA-COR cluster to act as a discrete guest for a much larger shell (vide infra).

The realization of the outermost shell is challenging because the diameter of the DBA-(4)-OC10-ISA-COR cluster is ca. $6.0 \mathrm{~nm}$ and thus it requires host network with relatively large cavities. Molecular models suggested that a DBA-OC26 derivative (Fig. 1a) with six $\mathrm{OC}_{26} \mathrm{H}_{53}$ chains can in principle serve as the outermost shell and provide the large cavities needed for trapping the DBA-(4)-OC10-ISA-COR cluster. Given the installation of six alkoxy chains, it is expected to form an extended honeycomb network with anticipated $p 6$ symmetry. The choice of DBAs as the constituents of outer shells is motivated from the fact that they form relatively flexible networks, the diameter of which can be readily tuned by changing the alkoxy chain length. However, in general, the formation of open porous networks with large cavities is energetically expensive and thus it remained to be seen whether DBA-OC26 can efficiently serve as the outermost shell in the anticipated concentric supramolecular network. ${ }^{17}$

The formation of an ordered monolayer comprised of COR-ISA clusters has been reported by us earlier. ${ }^{14}$ The monolayer is typically obtained by mixing an excess of COR with ISA solution in octanoic acid (OA). COR-ISA clusters are close-packed and interact with each other via van der Waals interactions (see Fig. S3 in ESI $\dagger$ ). The next step is the realization of the threecomponent network. The desired network was obtained by application of a drop of OA solution containing DBA-(4)-OC10 $\left(1.6 \times 10^{-6} \mathrm{M}\right)$, ISA $\left(2.2 \times 10^{-3} \mathrm{M}\right)$ and COR $\left(1.3 \times 10^{-4} \mathrm{M}\right)$ to the basal plane of freshly cleaved HOPG. In this network, six molecules of DBA-(4)-OC10 form a hexagonal shell around the COR-ISA heterocluster and these hexagonal heteroclusters are further packed in an ordered fashion. All the three-components are clearly resolved by STM (Fig. 2). The structure is stable to STM scanning and is imaged with high-resolution. At a different solution composition, significant co-adsorption of DBA-(4)-OC10 is observed, however the domains are phase separated from the three-component domains (see Fig. S4 in ESI $\dagger$ ).

Apart from the network described above, another threecomponent network is observed at compositions with higher COR concentration $\left(5.3 \times 10^{-4} \mathrm{M}\right)$, which differs in the number
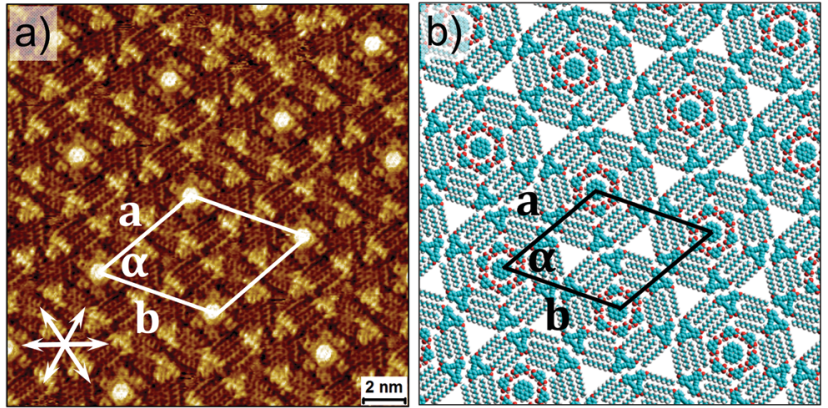

Fig. 2 (a) STM image showing the three-component network with twoconcentric shells obtained at the octanoic acid/HOPG interface. Solution composition [DBA-(4)-OC10 (0.012): ISA (16.9): COR (1.0)]. Graphite symmetry axes are shown in lower left corner. (b) Molecular model for the three-component system. The unit cell $\left(a=b=5.8 \pm 0.1 \mathrm{~nm}, \alpha=60 \pm 1^{\circ}\right)$ consists of 13 molecules: 6 DBA-(4)-OC10, 1 COR and 6 ISA. 


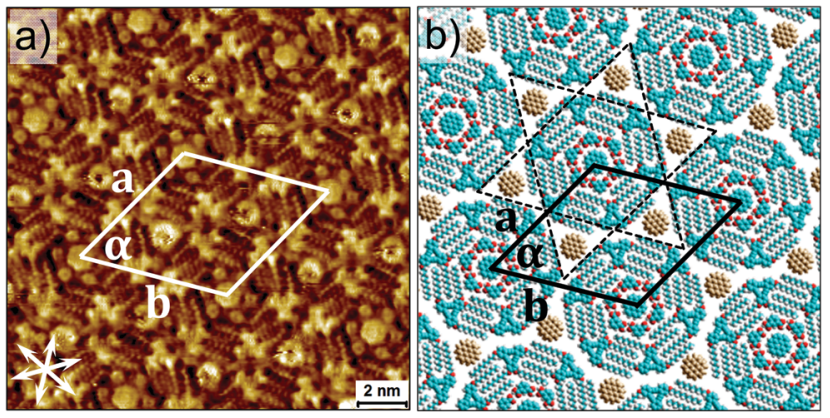

Fig. 3 (a) STM image showing the three-component Kagome network with two-concentric shells obtained at the OA/HOPG interface. Solution composition [DBA-(4)-OC10 (0.003): ISA (4.2): COR (1)]. Graphite symmetry axes are shown in lower left corner. (b) Molecular model for the threecomponent Kagomé system. The unit cell $\left(a=b=6.1 \pm 0.1 \mathrm{~nm}, \alpha=60 \pm 1^{\circ}\right)$ consists of 15 molecules: 6 DBA-(4)-OC10, 6 ISA and 3 COR. The COR molecules occupying the interstitial sites between DBA hexagons are shown in brown colour.

of COR molecules per unit cell. The hexagonal cavities are occupied by COR-ISA and additional COR molecules adsorb in the interstitial triangular cavities between adjacent DBA-(4)OC10-ISA-COR clusters giving rise to a Kagomé pattern (Fig. 3 and Fig. S5 in ESI $\dagger$ ). The observation of DBA-(4)-OC10-ISA-COR clusters separated by COR indicates that this three-component cluster is a robust supramolecular entity by itself.

The final step is the realization of extended four-component network. It was obtained by deposition of OA solution containing the four components namely, DBA-OC26 $\left(2.8 \times 10^{-6} \mathrm{M}\right)$, DBA-(4)$\operatorname{OC10}\left(3.2 \times 10^{-6} \mathrm{M}\right)$, ISA $\left(2.7 \times 10^{-4} \mathrm{M}\right)$ and COR $\left(2.3 \times 10^{-5} \mathrm{M}\right)$. Fig. 4a shows STM image of the four-component structure obtained at the OA/HOPG interface. It is clearly evident that the DBA-(4)-OC10-ISA-COR heterocluster is immobilized inside the DBA-OC26 cavities. Each DBA-OC26 cavity thus represents the outermost shell whereas the subsequent inner shells are formed by DBA-(4)-OC10 and ISA, respectively (see Fig. S6 in ESI $\dagger$ ).

While the design strategy appears to work in principle, the surface coverage of the four-component network is relatively poor. As evident from the STM image provided in Fig. 4a, the
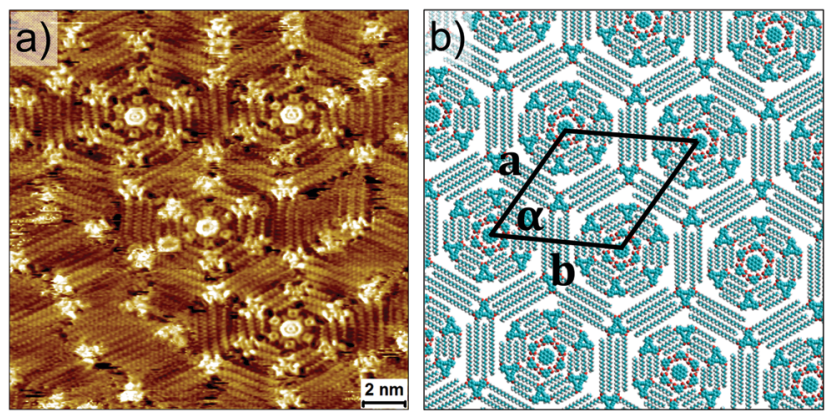

Fig. 4 (a) STM image of the four-component network with three-concentric shells obtained at the OA/HOPG interface. Solution composition [DBA-OC26 (0.12): DBA-(4)-OC10 (0.14): ISA (11.7): COR (1)]. (b) Molecular model for an ideal, extended network of the four-component system. The unit cell $\left(a=b=8.0 \pm 0.1 \mathrm{~nm}, \alpha=60 \pm 1^{\circ}\right)$ consists of 15 molecules: 2 DBA-OC26, 6 DBA-(4)-OC10, 6 ISA and 1 COR. four-component hexagons are surrounded by the densely packed network of DBA-OC26 (see Fig. S6 and S7 in ESI †). DBA derivatives are known to form densely packed structures at higher concentrations and the concentration range over which the porous to dense phase transition occurs depends on the length of the alkoxy chains: the longer the alkoxy chains, the lower the concentration at which the porous phase appears. ${ }^{17}$ Thus DBAs with significantly long alkoxy chains such as DBA-OC26 do not readily form porous networks. The porous network formation also depends on the solvent. While 1,2,4-trichlorobenzene (TCB) and 1-phenyloctane favours the formation of porous network, densely packed linear structures are preferentially formed from tetradecane and OA. ${ }^{18}$

DBA-OC26 alone does not form a porous network even at concentrations as low as $7.7 \times 10^{-7} \mathrm{M}$ at the OA/HOPG interface, which is an order of magnitude lower than the one used to obtain the four-component system. At this concentration, the surface coverage decreases drastically and only isolated domains of densely packed network are observed (see Fig. S8 in ESI $\dagger$ ). Thus the main roadblock lies in the concentration dependent phase behaviour of the DBA-OC26 system which allows formation of a structure other than the one anticipated from the design. The two- as well as three- component systems on the other hand, yield long-range ordered crystalline networks with relatively less defects. Annealing the sample as well as addition of excess DBA-(4)-OC10ISA-COR did not improve the surface coverage of the fourcomponent hexagons.

An important aspect that merits special attention for the successful realization of multicomponent supramolecular networks is competitive adsorption. ${ }^{6,19,20}$ Each molecule has different adsorption energy and the stabilization of a given component is also governed by the type of supramolecular interaction it has with its partner. The adsorption energies are typically compensated by adjusting the solution mole ratio. As a consequence, the solution composition which leads to formation of a given multicomponent system is rarely the same as expected from the stoichiometry deduced from an ideal surface-adsorbed network. This is reflected in the imperfect stoichiometric compositions used to obtain the multicomponent networks. The solution composition was typically arrived at based on purely empirical considerations and the compositions reported here are the ones that successfully yielded concentric supramolecular networks. This is in line with our previous observation that a perfect stoichiometric solution composition is not a pre-requisite for successful multicomponent self-assembly. ${ }^{6}$ Non-ideal surface compositions are observed in the present case, wherein phase-separated domains of another constituent component were found to co-exist with the expected multicomponent network is also a result of competitive adsorption.

While considering the few shortcomings discussed above one must bear in mind that this is a fairly complex system that comprises a total of six components (including the solvent and the substrate) thus leading to a complex scheme of interfacial and intermolecular interactions. It can be argued that the energy landscape of the system is shallow with several local minima. This brings us to the lingering conundrum about kinetics versus thermodynamics of self-assembly. While kinetic factors may have played role in the multicomponent assembly 
described above, there is sufficient indication that these networks are formed under thermodynamic control.

The first indication comes from the fact that all the shells (DBA-OC26, DBA-(4)-OC10 and ISA) are only formed in presence of an inner core (guest) component. Systematic control experiments revealed that concentration control does not yield porous networks for any of the shell components in absence of the guest species. ISA forms a densely packed zigzag network in absence of $\mathbf{C O R}^{21}$ (see Fig. S9 in ESI $\dagger$ ) whereas DBA-(4)-OC10 forms a relatively compact network (see Fig. S10 in ESI $\dagger$ ) in absence of the COR-ISA guest cluster. As discussed already, DBA-OC26 only forms hexagonal pores in response to the DBA-(4)-OC10-ISA-COR guest cluster. This multistep templating of outer shells by inner components is a unique feature of the concentric multicomponent assembly. ${ }^{22}$

The second aspect concerns the handedness of DBA shells (see Fig. S11 in ESI $\dagger$ ). It is evident from the analysis of STM images of the four-component system that the handedness of DBA shells, which is defined by the interdigitation pattern of the alkoxy chains, is often (92\%) the same for the inner (DBA-(4)-OC10) and the outer (DBA-OC26) shells indicating the transfer of chiral information between subsequent shells (see Fig. S12 in ESI $\dagger$ ). Last but not the least, when the inner DBA-(4)-OC10 shell is distorted, the outer DBA-OC26 shell also shows distorted pattern in the alkoxy chain interdigitation (see Fig. S12 in ESI $\dagger$ ). All the factors described above strongly indicate that the guest structures are not merely kinetically trapped in host networks but are formed as a result of a hierarchical molecular recognition process wherein there is active transfer of structural information between assembling components.

In conclusion, the work described above introduces the concept of concentric multicomponent self-assembly on solid surfaces. The design strategy based on size and shape complementarity is effective for construction of the anticipated four-component cluster. Realization of large area, long-range ordered networks of the four-component clusters however proved to be challenging and is complicated by the phase behavior of the molecule that constitutes the outermost shell. The guest (clusters) template the formation of successive shells. The concentric shells undergo a role reversal in a hierarchical fashion and are encapsulated by a larger supramolecular ring. The efficiency of molecular recognition that underpins the templating behavior decreases after addition of the second shell. The results described above are promising for developing alternative strategies for construction of sophisticated multicomponent networks.
G. V. acknowledges award of Marie Sklodowska-Curie individual fellowship (number 706314, GRAPHIL). This work is supported by the Fund of Scientific Research-Flanders (FWO), KU Leuven Internal Funds, Belgian Federal Science Policy Office (IAP-7/05), European Research Council under the European Union's Seventh Framework Programme (FP7/2007-2013)/ERC Grant Agreement No. 340324 and JSPS KAKENHI Grant Numbers 15H02164 and 26620063.

\section{Notes and references}

1 A. G. Slater, L. M. A. Perdigão, P. H. Beton and N. R. Champness, Acc. Chem. Res., 2014, 47, 3417-3427.

2 T. Kudernac, S. Lei, J. A. A. W. Elemans and S. De Feyter, Chem. Soc. Rev., 2009, 38, 402-421.

3 M. Surin and P. Samori, Small, 2007, 3, 190-194.

4 J. Liu, T. Chen, X. Deng, D. Wang, J. Pei and L.-J. Wan, J. Am. Chem. Soc., 2011, 133, 21010-21015.

5 K. Cui, F. Schlütter, O. Ivasenko, M. Kivala, M. G. Schwab, S.-L. Lee, S. F. L. Mertens, K. Tahara, Y. Tobe, K. Müllen, K. S. Mali and S. De Feyter, Chem. - Eur. J., 2015, 21, 1652-1659.

6 J. Adisoejoso, K. Tahara, S. Okuhata, S. Lei, Y. Tobe and S. De Feyter, Angew. Chem., Int. Ed., 2009, 48, 7353-7357.

7 J. A. Theobald, N. S. Oxtoby, M. A. Phillips, N. R. Champness and P. H. Beton, Nature, 2003, 424, 1029-1031.

8 K. Tahara, K. Kaneko, K. Katayama, S. Itano, C. H. Nguyen, D. D. D. Amorim, S. De Feyter and Y. Tobe, Langmuir, 2015, 31, 7032-7040

9 J. Teyssandier, S. D. Feyter and K. S. Mali, Chem. Commun., 2016, 52, 11465-11487.

10 Y. Xue and M. B. Zimmt, J. Am. Chem. Soc., 2012, 134, 4513-4516.

11 A. Bhattarai, U. Mazur and K. W. Hipps, J. Am. Chem. Soc., 2014, 136, 2142-2148.

12 U. Mazur and K. W. Hipps, Chem. Commun., 2015, 51, 4737-4749.

13 R. Gutzler, L. Cardenas and F. Rosei, Chem. Sci., 2011, 2, 2290-2300.

14 S. Lei, M. Surin, K. Tahara, J. Adisoejoso, R. Lazzaroni, Y. Tobe and S. D. Feyter, Nano Lett., 2008, 8, 2541-2546.

15 S. J. H. Griessl, M. Lackinger, F. Jamitzky, T. Markert, M. Hietschold and W. M. Heckl, Langmuir, 2004, 20, 9403-9407.

16 S. Lei, K. Tahara, J. Adisoejoso, T. Balandina, Y. Tobe and S. De Feyter, CrystEngComm, 2010, 12, 3369-3381.

17 S. Lei, K. Tahara, F. C. De Schryver, M. van der Auweraer, Y. Tobe and S. De Feyter, Angew. Chem., Int. Ed., 2008, 47, 2964-2968.

18 K. Tahara, S. Furukawa, H. Uji-i, T. Uchino, T. Ichikawa, J. Zhang, W. Mamdouh, M. Sonoda, F. C. De Schryver, S. De Feyter and Y. Tobe, J. Am. Chem. Soc., 2006, 128, 16613-16625.

19 K. Kim, K. E. Plass and A. J. Matzger, J. Am. Chem. Soc., 2005, 127, 4879-4887.

20 M. Hibino, Langmuir, 2016, 32, 4705-4709.

21 M. Lackinger, S. Griessl, T. Markert, F. Jamitzky and W. M. Heckl, J. Phys. Chem. B, 2004, 108, 13652-13655.

22 S. A. L. Rousseaux, J. Q. Gong, R. Haver, B. Odell, T. D. W. Claridge, L. M. Herz and H. L. Anderson, J. Am. Chem. Soc., 2015, 137, 12713-12718. 